\title{
Utilising social media to educate and inform healthcare professionals, policy-makers and the broader community in evidence-based healthcare
}

\author{
Amy Keir, ${ }^{1,2}$ Nicolas Bamat, ${ }^{3}$ Ravi Mangal Patel, ${ }^{4}$ \\ Omar Elkhateeb, ${ }^{5}$ Damian Roland ${ }^{6,7}$
}

10.1136/bmjebm-2018-111016

${ }^{1}$ Robinson Research Institute and the Adelaide Medical School, University of Adelaide, North Adelaide, South Australia, Australia ${ }^{2}$ Healthy Mothers, Babies and Children Theme, South Australian Health and Medical Research Institute, North Adelaide, South Australia, Australia

${ }^{3}$ Division of Neonatology, Center for Pediatric Clinical Effectiveness, The Children's Hospital of Philadelphia, Philadelphia, Pennsylvania, USA

${ }^{4}$ Emory University School of Medicine, Atlanta, Georgia, USA

${ }^{5}$ King Fahad Medical City, Riyadh, Saudi Arabia ${ }^{6}$ Paediatric Emergency Medicine Leicester Academic (PEMLA) Group, Leicester Royal Infirmary, Leicester, UK ${ }^{7}$ Department of Health Sciences, SAPPHIRE Group, Leicester University, Leicester, UK

Correspondence to: Dr Amy Keir, Women's and Children's Hospital, North Adelaide SA 5006, Australia; amy.keir@adelaide.edu.au

\section{Aheck for updates}

( $)$ Author(s) (or their employer(s)) 2019. No commercial re-use. See rights and permissions. Published by BMJ.

To cite: Keir A, Bamat $\mathrm{N}$, Patel RM, et al. BMJ

Evidence-Based Medicine 2019;24:87-89.
Social media is emerging as key solution to increase collaborative discourse between individuals, institutions and countries. Although evidence of social media's impact on health policy is limited, ${ }^{1}$ its potential to promote knowledge dissemination and provide open forums for critical appraisal of evidence-based literature is increasingly clear. ${ }^{2}$ Social media in many ways is the definition of dissemination. It can be an active tool for spreading evidence-based information to a target audience (population) via determined channels (social media platforms) using planned strategies. Social media has a heterogeneous array of definitions as it can describe particular platforms of use (ie, Twitter or Facebook) or a particular methodology of connecting users. Social media in medicine can be defined as any digital media that enables widespread connectivity between users using a defined methodology of approach (ie, blog, podcast and so on). ${ }^{1}$

Researchers in the field of social media and knowledge dissemination in healthcare have clearly put it 'Let debates take place in open and public forums, rather than the isolated circles of individual clinicians or local groups. Engagement and participation can usher in a new era of transparency around clinical decision-making, knowledge integration, and evidence-based practice. ${ }^{2}$ The Evidence Manifesto $^{3}$ was conceived, with similar ideals, to address the growing challenge of integrating patient-centred decision making with the best available evidence: 'Informed decision making requires clinicians and patients to identify and integrate relevant evidence. But with the questionable integrity of much of today's evidence, the lack of research answering questions that matter to patients, and the lack of evidence to inform shared decision how are they expected to do this?'3 One of the key steps identified in the Evidence Manifesto was to 'Educate professionals, policy-makers and the public in evidence-based healthcare to make informed choices. ${ }^{3}$ There is no one best way to do this. In fact, policies that approach this issue through one methodology are likely to fail. One medium that is growing in academic activity and engagement is social media, including blogs, forums and social networks.

The ability to objectively determine the impact of social media on knowledge translation is challenging. However, there are ways to assess the quality of social media research, ${ }^{4}$ the quality of social media modalities such as blogs and podcasts ${ }^{5}$ and the potential impact of social media on dissemination of knowledge $^{67}$ and case studies of the impact of social media on knowledge translation exist. As social media becomes increasingly accepted by the healthcare professional community, new terminology must be described and defined. For example, networks such as Twitter or Facebook need to be differentiated from specialist search and analysis engines such as Symplur or Semantria. The former being online platforms which deliver a distinct method of connectivity between users (the character limit of twitter being a popular example) and the latter being specific algorithms which analyse data on, or between, these platforms [figure 1]. This analysis may be a simple quantitative description of the number of times a particular hashtag is cited or more detailed descriptions of the tone of a particular sequence of social media activity. For example, sentiment analysis can determine if activity around a particular topic is positive or negative.

A successful example of social media use is the free open-access medical education (FOAM) international movement that has brought together people from many backgrounds and specialties. ${ }^{8}$ FOAM has been described as a 'dynamic collection of resources and tools for lifelong learning in medicine, as well as a community and an ethos'. ${ }^{8}$ It exists almost entirely on social media through websites, Twitter and other online platforms. The use of FOAM has made it easier for healthcare professionals to interact with colleagues from around the world to discuss new research, share and develop evidence-based medicine resources. ${ }^{10}$ Social media use, like the FOAM movement, is associated with the development of communities of practice that promote the transfer of information at scale, crossing institutional and geographical boundaries not previously possible. These forums also allow shared conversations around critical appraisal of new evidence that is transparent and accessible to users.

We have attempted to do this in neonatology with the formation of a Community of Practice ${ }^{11}$ consisting of providers dedicated to leveraging social media for the benefit of evidence-based 


\section{NOECO}

A framework for reporting research in healthcare social media The PICO for Networks

\section{₹ Network}

Example: Twitter or Facebook

\section{Q Object}

Example: Hasthtags or post sentiment analysis

\section{Engine}

Example: Symplur or Semantria

\section{$\Delta \Delta$ Comparison}

Example: Regular distribution vs. clustering in Twitte or Sentiment change before and after an event

\section{Observation}

Example: Twitter users are clustered around few nodes or sentiment in a community changed after an event

\section{Roland, Spurr \& Cabrera WestJEM}

Figure 1 A framework for reporting research in healthcare social media Reused in an unedited format under a Creative Commons Attribution 4.0 International (CC BY 4.0) License.

medicine. This community was formalised when neonatologists and a paediatric emergency physician who use the social media platform Twitter to disseminate new research findings met at the Paediatric Academic Societies meeting this year in Toronto. The meeting idea originated on Twitter and was coordinated through the same platform. At the gathering, the hashtag \#neoEBM was born as way to disseminate and track evidence-based neonatology content. ${ }^{12}$ While in its infancy, we hope that we can build on the links already formed and strengthen our group to provide trustworthy and insightful debate in evidence-based neonatal medicine. This emerging community has already opened up collaborations between individuals, various evidence-based neonatal practice groups and journals. In just under 2 months since its inception in May 2018 \#neoEBM appeared in 1925 posts from 502 user accounts, garnering 2.5 million impressions on Twitter alone.

The use of social media to disseminate evidence-based healthcare perhaps represents one of the key tools of the future generation of evidence-based medicine leaders. ${ }^{3}$ To strengthen this tool, we need broader engagement and use as well as collaboration, coordination and guidance towards effective dissemination strategies. We need online sources and experts that we can trust to deliver evidence-based healthcare information, using critical appraisal in discussion of new research findings. We also need research to understand the impact on clinical practice of social media as a dissemination tool of evidence-based medicine. Biomedical journals are also moving toward social media to disseminate content, ${ }^{13}$ although with mixed results. ${ }^{14}$
There are potential challenges, however, around the growing influence of social media in healthcare and research. Questions around the use of social media to disseminate evidence-based healthcare information currently subject to ongoing research and debate include:

1. What are the most effective ways of disseminating evidencebased healthcare information on social media?

2. How can trustworthy sources of social media be developed, coordinated and maintained to deliver information on evidence-based healthcare?

3. Is editorial oversight possible on online platforms?

4. How can we effectively involve patient and family advocacy groups in social media platforms to improve dissemination of evidence-based healthcare information and support shared-decision making?

5. How do we ensure that healthcare professionals, researchers, policy-makers, patients and families have the skills to determine what are trustworthy information sources derived from social media?

6. How do we create a safe environment for communication through social media between healthcare providers and the broader community?

We propose that social media can be a valuable tool to disseminate knowledge around evidence-based healthcare, including new research findings and critical appraisal of current practice. We look forward to the academic and clinical community, as well as patients and families and policy-makers, contributing to answering these questions.

\section{Collaborators Brett Manley.}

Contributors All authors contributed to the concepts behind the article. AK: wrote the initial draft. NS, RMP, OE and DR: provided additional input, critically appraised and further contributed to the writing of the paper.

Competing interests None declared.

Provenance and peer review Not commissioned; externally peer reviewed.

\section{References}

1. Roland D. Social Media, Health Policy, and Knowledge Translation. J Am Coll Radiol 2018;15:149-52.

2. Chan T, Trueger NS, Roland D, et al. Evidence-based medicine in the era of social media: Scholarly engagement through participation and online interaction. CJEM 2018;20:3-8.

3. Heneghan C, Mahtani KR, Goldacre B, et al. Evidence based medicine manifesto for better healthcare. BMJ 2017;357:j2973.

4. Roland D, Spurr J, Cabrera D. Integrating Emergency Care with Population Health. Initial Standardized Framework for Reporting Social Media Analytics in Emergency Care Research: Western Journal of Emergency Medicine, 2018.

5. Paterson QS, Thoma B, Milne WK, et al. A Systematic review and qualitative analysis to determine quality indicators forhealth professions education blogs and podcasts. J Grad Med Educ 2015;7:549-54.

6. Thoma B, Chan TM, Paterson QS, et al. Emergency medicine and critical care blogs and podcasts: establishing an international consensus on quality. Ann Emerg Med 2015;66:396-402.

7. Thoma B, Sanders JL, Lin M, et al. The social media index: measuring the impact of emergency medicine and critical care websites. West J Emerg Med 2015;16:242-9.

8. Nickson CP, Cadogan MD. Free Open Access Medical education (FOAM) for the emergency physician. Emerg Med Australas 2014;26:76-83.

9. Cadogan M, Thoma B, Chan TM, et al. Free Open Access Meducation (FOAM): the rise of emergency medicine and critical care blogs and podcasts (2002-2013). Emerg Med J 2014;31(e1):e76-e77. 
10. Roland D, Spurr J, Cabrera D. Preliminary evidence for the emergence of a health care online community of practice: Using a netnographic framework for twitter hashtag analytics. J Med Internet Res 2017;19:e252.

11. Lave J, Wenger E. Situated learning: Legitimate peripheral participation. Cambridge: Cambridge University Press, 1991.

12. Evidence-Based Neonatology [EBNEO]. ALERT!! This group of devotees converged and recommended: using \#NeoEBM as an umbrella term to tag content for the global community interested in promoting evidence- based neonatal practice, and \#foamneo to tag free-open access content. We'll try to adhere- let's see if it sticks! [Tweet. https://twitter.com/ EBNE0/status/993261436959887365 (6 May 2018).

13. Thoma B, Murray H, Huang SYM, et al. The impact of social media promotion with infographics and podcasts on research dissemination and readership. CJEM 2018;20:300-6.

14. Fox CS, Bonaca MA, Ryan JJ, et al. A randomized trial of social media from Circulation. Circulation 2015;131:28-33. 